\title{
NUMERICAL SIMULATION OF METHANE UNDER PHASE TRANSFER CONDITIONS WITH ITS REAL PROPERTIES
}

\author{
Boris V. Borisov ${ }^{1, \text { a }}$, Sergey S. Bondarchuk ${ }^{2}$, Dimitrii O. Skryabinsky ${ }^{1}$, Victorya S. Striha ${ }^{1}$ \\ ${ }^{1}$ National Research Tomsk Polytechnic University, 634050 Tomsk, Russia \\ ${ }^{2}$ National Research Tomsk State University, 634050 Tomsk, Russia
}

\begin{abstract}
The technique and results of calculation of parameters of the flow of methane in single and two-phase state is discussed. The flow is described by the one-dimensional nonstationary equations of a continuous medium. Is taken into account the processes of friction, heat transfer and phase transitions. The results of the application of various schemes of numerical integration of the equations are discussed.
\end{abstract}

In this paper the parameters of the flow of metal having generally two phases investigates. For a description of the thermodynamic properties of the working wide range of parameters an approximation of the experimental data suggested in the article [1] in a fluid equation of state is used. The essence of this approach lies in the fact that the experimental data for methane were approximated by the authors for the molar Helmholtz free energy, as well as approximating were chosen easily integrable and is easily differentiable functions.

The parameters are determined by the current system of equations [2]:

$$
\begin{gathered}
\frac{\partial \rho}{\partial \tau}+\frac{\partial \rho u}{\partial x}=-G_{\text {condensate }}, \\
\frac{\partial \rho w}{\partial \tau}+\frac{\partial\left(\rho u^{2}+p\right)}{\partial x}=-F_{\text {friction }}-G_{\text {condensate }} \cdot w, \\
\frac{\partial \rho\left(\varepsilon+0.5 \cdot u^{2}\right)}{\partial \tau}+\frac{\partial \rho u\left(h+0.5 \cdot u^{2}\right)}{\partial x}=-Q_{\text {out }}-G_{\text {condensate }} \cdot h_{\text {condensate }},
\end{gathered}
$$

Where $\tau, x, \rho, u, p, \varepsilon, h, G_{\text {condensate }}, F_{\text {friction }}, Q_{\text {out }}$, respectively, time, coordinate, density, velocity, pressure, specific internal energy, specific enthalpy, mass flow rate of the phase transition from the condensation on the walls, or evaporation from the surface of the film of condensation on the walls of the channel, the force of friction and heat flux. In this case $G_{\text {condensate }}, F_{\text {friction }}, Q_{\text {out }}$ given on unit volume.

\section{a Corresponding author: bvborisov@tpu.ru}

This is an Open Access article distributed under the terms of the Creative Commons Attribution License 4.0, which permits unrestricted use, distribution, and reproduction in any medium, provided the original work is properly cited. Article available at nttp://www.epl-conterences.org or nttp://dx.dol.org/10.1051/ep|cont/201617001011 
The initial conditions are given a uniform initial distribution of density, velocity and internal energy as the rest gas, and for uniformly moving stream for whole canal.

Border conditions:

$$
G(0, \tau)=G_{0}(0) ; \quad h(0, \tau)=h_{0}(0) ; \quad p(l, \tau)=p_{0}(\tau)
$$

System (1) - (4) together with the state equation is solved numerically. Using of equation of state is implemented as a procedure, an algorithm which allows for the input parameters of the density and specific internal energy, found from the system of equations (1) - (3), determine the values of the appropriate temperature and then all the other thermodynamic parameters [1], used for numerical integration of (1) - (3).

Simulation is performed with taking into account of the hydraulic and thermal interaction with the walls of the tube. For this the numerical implementation of the control (finite) volume method has been selected [2]. To determine the greatness of the original stream was used TVD scheme with approximation of derivatives against the flow and using the principle of the minimum of the derivative. The use of this approach was followed by the appearance of significant instability that is a consequence of a very strong dependence of the pressure and specific internal energy according to the equation of state. The introduction of artificial viscosity in the flow of the total momentum and the total enthalpy in the form proposed in 1944 and von Neumann J. Richtmyer R.D. [2] only partly solved the problem. Scheme proposed by Lax P.D. in 1954 [2] for numerical integration helped fully repay nonphysical oscillation of solutions. The very limited number of Courant was used. This scheme has a substantial viscosity, which allows it to avoid significant fluctuations nonphysical solutions. But some embodiments, the calculation results included an unjustified increase in the pressure in the steady stream through the channel of constant cross section with increasing density and fall of the speed, in some cases, when a two-phase flow simulation included on hydraulic losses and heat exchange with the wall of the channel for providing condensation conditions.

Example of a detailed calculation for the steady flow is shown in Figure 1.

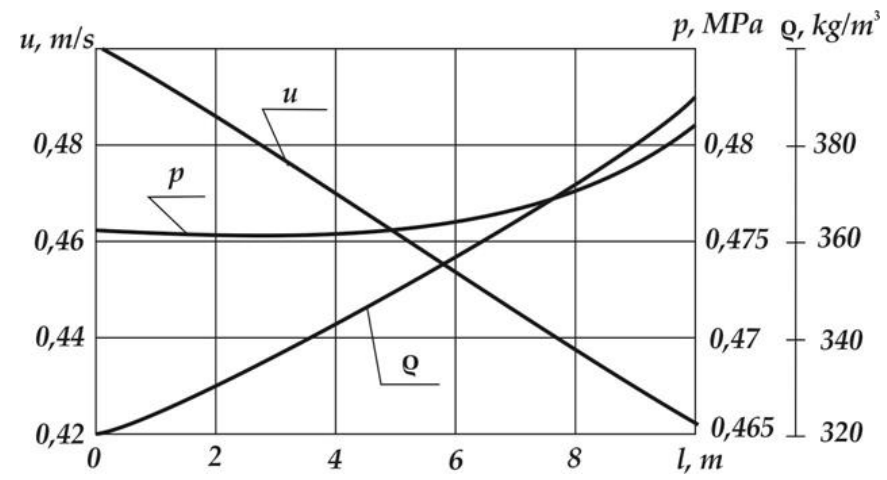

Figure 1. Parameters in the two-phase flow field of methane produced by the scheme Lax PD.

This the pressure behavior is explained due to on the one hand the strong diffusion properties of Lax's schemes, and on the other hand, the special features determining the pressure according to of the obtained values of density and internal energy [1]. Authors have the experience the use for determine of gas-dynamic parameters in difficult areas and in conditions of high gradients of pressure due to monotone SK Godunov scheme of decay of a discontinuity [3, 4]. This method of determining the values of flows calculated on the channel border and at the borders of cells is well physically justified. For an ideal gas, this method is deeply studied and widely used.

As for implementation for a real gas of such an algorithm is difficult. Based on the proposal of SK Godunov [3] trusts that the flow does not contain strong discontinuities parameters. In this case the shock waves and the waves underpressure converted to sound waves. Dynamic relations on the 
discontinuities derived from the equations of continuity, momentum and energy (1-3) are determined by [3]:

$$
\begin{aligned}
& {[\rho] \mathrm{D}-[\rho u]=0,} \\
& {[\rho u] \mathrm{D}-\left[p+\rho u^{2}\right]=0,} \\
& {\left[\rho\left(\varepsilon+0.5 \cdot u^{2}\right)\right] D-\left[\rho u\left(\varepsilon+p / \rho+0.5 \cdot u^{2}\right]=0 .\right.}
\end{aligned}
$$

It is believed that the mass flow rate of gas through the discontinuity $a_{i}$ is determined by the relation $a_{i}=\rho_{i} c_{i}$, and the speed of propagation of perturbations $D_{i}$ (for "right" and "left" characteristics, respectively) is given by: $D_{i}=w_{i} \pm c_{i}$.

To determine the flow variables are considered four options presented schematically in Figure 2. In these schemes shows propagating discontinuities and contact discontinuity according change in the $\mathrm{x}$ and in the time relative to the boundary $(x=0)$ between the cells named Latin numbers.

A)

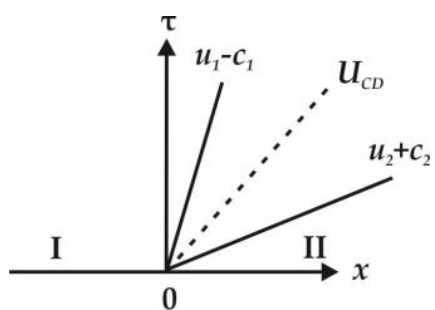

C)

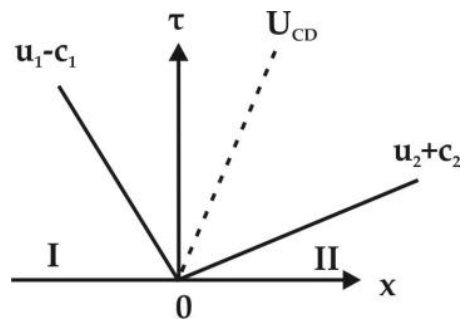

B)

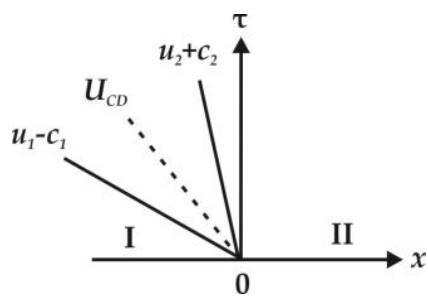

D)

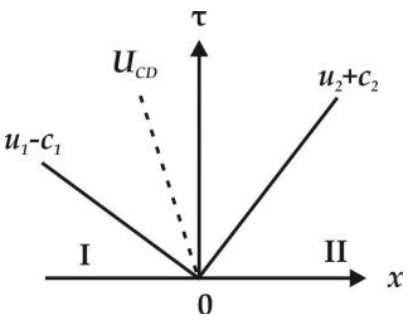

Figure 2. Schemes options decay of an arbitrary discontinuity.

Density $R o$, pressure $P$, velocity $U$ and specific energy $E$ at the interface between the cell is determined according to the following analysis of the decay of an arbitrary discontinuity options:

Option (A): $\left(u_{1}-c_{1}\right) \geq 0$. There is the supersonic flow "to right". Parameters at the cell border assumed equal the parameters of the left cell: $R o=\rho_{1}, U=u_{1}, P=p_{1}, E=\varepsilon_{1}$.

Option (B): $\left(u_{2}+c_{2}\right) \leq 0$. There is the supersonic flow "to left". Parameters at the cell border assumed equal the parameters of the cell right: $R o=\rho_{2}, U=u_{2}, P=p_{2}, E=\varepsilon_{2}$.

To analyze of the two other options the speed and pressure of the contact discontinuity is determined according to by SK Godunov proposal [3]:

$$
U_{C D}=\frac{a_{1} u_{1}+a_{2} u_{2}+p_{1}-p_{2}}{a_{1}+a_{2}},
$$

and 


$$
P_{C D}=\frac{p_{1} a_{2}+p_{2} a_{1}+\left(u_{1}-u_{2}\right) a_{1} a_{2}}{a_{1}+a_{2}}
$$

Option (C): $\left(u_{1}-c_{1}\right)<0,\left(u_{2}-c_{2}\right)>0$ and $U_{C D}>0$. The density and internal energy is determined from (5) - (7) according to the "left" elementary wave:

$$
R o=\frac{a_{1}}{\left(c_{1}-u_{1}\right)+U_{C D}}, E=\frac{a_{1}\left(\varepsilon_{1}+0.5 \cdot u_{1}^{2}\right)-p_{1} u_{1}+P_{C D} U_{C D}}{R o \cdot\left(u_{1}-c_{1}+U_{C D}\right)}-\frac{U_{C D}^{2}}{2} .
$$

Option (D): $\left(u_{1}-c_{1}\right)<0,\left(u_{2}-c_{2}\right)>0$ and $U_{C D}<0$. The density and internal energy is determined from (5) - (7) according to the "right" elementary wave:

$$
R o=\frac{a_{2}}{\left(c_{2}-u_{2}\right)+U_{C D}}, E=\frac{a_{2}\left(\varepsilon_{2}+0.5 \cdot u_{2}^{2}\right)-p_{2} u_{2}+P_{C D} U_{C D}}{R o \cdot\left(u_{2}-c_{2}-U_{C D}\right)}-\frac{U_{C D}^{2}}{2} .
$$

The solutions of elementary waves are used to implement the boundary conditions in accordance with the sign of the velocity at the boundary. It is believed that the channel is finished at borders of the large volumes, which are having a complete set of relevant thermodynamic parameters.

For definiteness we consider the ratio of parameters on the left border. It is believed that the flow is carried out in the subsonic mode, and in accordance with the pressure at the corresponding pressure in the volume $\left(P=p_{0}\right)$.Speed and density determined by the decision on the appropriate wave: $U=u_{2}+\frac{P-p_{2}}{\rho_{2}}$ and $R o=\frac{a_{2}{ }^{2}}{a_{2} c_{2}-\left(P-p_{1}\right)}$. In case inflow $(U>0)$ considered that the specific enthalpy corresponds to a value in the adjacent volume and in case outflow $(U<0)$ the specific internal energy's value is determined according to the following (5) - (7) the relation:

$$
E=\frac{a_{2}\left(\varepsilon_{2}+0.5 \cdot u_{2}^{2}\right)+P U-p_{2} u_{2}}{\operatorname{Ro}\left[U-\left(u_{2}+c_{2}\right)\right]}-\frac{U^{2}}{2}
$$

Thanks to the SK Godunov circuit application and the appropriate implementation of the boundary conditions is received significant impact to the calculation of the parameters (Fig. 3) obtained in the same option of that the respective calculation of Lax PD (Fig. 1).

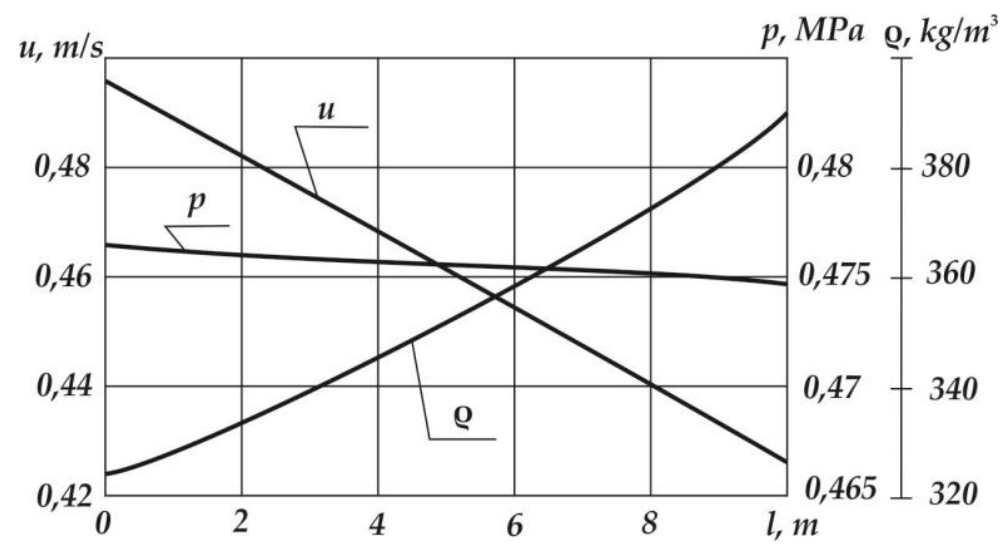

Figure 3. Parameters in the two-phase flow field of methane produced by the scheme Godunov SK. 
The presented method of calculation can be recommended for use in the process design of the special devices.

\section{References}

1. Daniel G. Friend, James F. Ely, and Hepburn Ingharn, Thermophysical properties of methane. - National Institute of standards and technology (Boulder, Colorado 1988)

2. P. J. Roache. Computational fluid dynamics. Albuquerque (Hermosa Publs, 1976)

3. S.K. Godunov,

A.V. Zabrodin,

M.Ya. Ivanov,

A.N. Kraiko, G.P. Prokopov. Chislennoe reshenie mnogomernykh zadach gazovoi dinamiki (Numerical Solution of Multidimensional Problems of Gas Dynamics, Moscow: Nauka, 1976)

4. S.S. Bondarchuk, A.B. Vorozhtsov, A.S. Zhukov, B.V. Borisov, Russian Physics Journal, 57 (12), 1796 (2015) 